\title{
Decontamination methods of personal protective equipment for repeated utilization in medical/surgical settings
}

\author{
Abdurrahman Kharbat BSA, Adin Mizer BS, Mimi Zumwalt MD
}

\begin{abstract}
The COVID-19 pandemic has affected citizens and healthcare workers worldwide due to a number of important factors. The transmission of the SARS CoV-2 microorganism, the pathogen that causes COVID-19 infection, occurs through droplet and aerosol spread due to coughs and sneezes from infected patients. A panicked public began hoarding medical supplies and personal protective equipment (PPE), leaving healthcare workers to care for patients without adequate protection. A literature review was conducted to better understand the options available to hospital and healthcare system administrators as they develop necessary protocols for the conservation and possible reuse of PPE. This review is based upon the peer-reviewed studies of various scientific investigators, biotechnology researchers, governmental agency health officials, including meta-analyses, preliminary/pilot studies, and policy statements. Current findings indicate that extended usage of $N 95$ respirators is practical since there are methods available for the decontamination/repeated use of N95 respirators. In evaluating the efficacy of such methods, the safety of healthcare workers is important in deciding which method to recommend. Available evidence supports the use of the Bioquell Hydrogen Peroxide Vapor (HPV) system for decontaminating N95 respirators. Information on other PPE will also be discussed about more specific items. Informed decisions regarding the policies of hospitals and healthcare systems must be considered, and with the safety of healthcare workers in mind, both factors influenced the recommendations made in this comprehensive review.
\end{abstract}

Keywords: coronavirus 2, COVID19, personal protective equipment, decontamination

\section{INTRODUCTION}

Rationale and Objectives: At the end of 2019 and the beginning of 2020, the world experienced a novel coronavirus, soon named SARS Coronavirus 2 (SARS CoV-2), which causes COVID-19 infection in humans. Symptoms of this coronavirus are similar to those brought on by other viruses of the same family, which include but are not limited to respiratory distress, fever, and a dry cough. The response

Corresponding author: Mimi Zumwalt

Contact Information: Mimi.zumwalt@ttuhsc.edu

DOI: $10.12746 /$ swrccc.v8i34.677 to the COVID-19 pandemic by governments around the world has been deemed weak and ineffectual; healthcare systems globally are overwhelmed by the exponential rise of infected patients. Most worrisome is the scarcity of resources such as hospital beds, ventilators, and personal protective equipment (PPE). PPE refers to a broad class of items worn to protect against/prevent exposure to biohazards and includes gloves, surgical masks (one-size-fit, no tight seal), respirators (fitted, tight seal), goggles/protective eyewear, face shields, gowns, plus other attire/clothing that provide patients and healthcare workers with a protective layer against communicable pathogens. Many of the PPE items are intended to be used simultaneously to afford maximum protection. With PPE shortages, 
it is important to note that certain types of PPE do not adequately replace the use of others; for example, a face shield does not protect against airborne pathogens as well as a respirator. Therefore, face shields should not be considered a replacement for respirators. ${ }^{1}$ Surges of panic, uncertainty, and misinformation have led to non-medical personnel stockpiling limited and essential PPE supplies, which has led to a dramatic shortage in many hospitals and healthcare systems. To this end, a systematic review of the literature has been conducted in search of alternative solutions to standard PPE, primarily evaluating the efficacy of home-made/Do-it-yourself (DIY) masks and/or using disinfected/reused masks as safe and plausible PPE alternatives. The efficacy of using various household materials in homemade/DIY masks will be evaluated on their ability to provide filtration efficiencies comparable to standard surgical masks; the effectiveness of various disinfection methods will also be discussed.

\section{Methods}

PubMed and Google Scholar were used to search for scientific studies in peer-reviewed journals with relevant keywords: PPE decontamination, N95 respirators, and alternative materials PPE. Data from policy briefs and studies from organizations such as the CDC were also compiled. These sources can be classified into three categories: meta-analysis, preliminary/pilot quantitative, and protocol/policy recommendations. Although no one source completely covers every element of the following criteria, these guidelines were helpful in establishing the integrity and relevance of available sources/cited literature:

1. The source presented relevant information about a subtopic of the field of study.

2. The source provided a previously unexplored perspective on an established policy or intervention.

3. The source focused on elements within the study of PPE use and decontamination methodologies.

4. The source is published in a peer-reviewed journal, by a governmental agency, or by a well-reputed non-governmental organization.

\section{RESULTS}

\section{DeContamination Methods}

\section{Hydrogen Peroxide Vapor}

Brooks et al prepared a report in July 2016 by Battelle, a not-for-profit applied science and technology development company, aimed to explore the efficacy of HPV decontamination of a selected N95 filtering facepiece respirator (FFR) and to characterize the impact of HPV on the mechanical integrity and performance of the FFR. The FFR used was an N95 FFR, Model 1860 (3M, St. Paul, MN). This project was undertaken in three phases: 1) determination of decontamination parameters, 2) impact of repeat decontamination cycles on functional performance of the FFR, and 3) assessment of repeated decontamination efficacy for up to 50 decontamination cycles. ${ }^{2}$

Phase I decontamination parameters were established to ensure a 6-log reduction in organism viability. This was achieved by inoculating swatches of FFR material with liquid droplets containing G. stearothermophilus spores, specifically selected due to the bacteria's known resistance to HPV decontamination. The loading bacteria quantity used was $1 \times 10^{6}$ colony forming units (CFU) per swatch, in order to fulfill a 6-log reduction upon complete inactivation of the organism, as seen in Table 1. Swatches were entered into the HPV cycle and removed at regular intervals then assayed to determine the exposure time needed to achieve complete inactivation. The final HPV cycle consisted of a 10minute conditioning phase, a 20-minute gassing phase at $2 \mathrm{~g} / \mathrm{min}$, a $150-$ minute dwell phase at $0.5 \mathrm{~g} / \mathrm{min}$, and a 300-minute aeration phase to achieve an undetectable level of hydrogen peroxide gas (achieve a point of no "off-gassing") on the FFR. Total cycle duration was 480 minutes (8 hours), with a hydrogen peroxide permissible exposure limit (PEL) of 1 ppm at 210 minutes, indicating that a shorter cycle time can be used. ${ }^{2}$

Phase II involved the evaluation of the mechanical integrity and performance of an FFR following 50 cycles of HPV decontamination; the performance tests used included inert aerosol collection efficiency, biological aerosol collection efficiency, inhalation resistance, and respirator fit on a mannequin mimicking the human 
Table 1. Summary of Decontamination Results Following Aerosol Inoculation with G. stearothermophilus

\begin{tabular}{|c|c|c|c|c|c|c|c|c|}
\hline Trial & HPV FFRs & Day 4 & Day 7 & $\begin{array}{l}\text { Streak } \\
\text { Plate }\end{array}$ & $\begin{array}{c}\text { Control } \\
\text { FFRs }\end{array}$ & Day 4 & Day 7 & $\begin{array}{l}\text { Streak } \\
\text { Plate }\end{array}$ \\
\hline \multirow{5}{*}{1} & $\# 1$ & Negative & Negative & Negative & $\# 1$ & Positive & Positive & Positive \\
\hline & $\# 2$ & Negative & Negative & Negative & $\# 2$ & Positive & Positive & Positive \\
\hline & $\# 3$ & Negative & Negative & Negative & $\# 3$ & Positive & Positive & Positive \\
\hline & $\# 4$ & Negative & Negative & Negative & $\# 4$ & Positive & Positive & Positive \\
\hline & $\# 5$ & Negative & Negative & Negative & $\# 5$ & Positive & Positive & Positive \\
\hline \multirow{5}{*}{2} & $\# 1$ & Negative & Negative & Negative & $\# 1$ & Positive & Positive & Positive \\
\hline & $\# 2$ & Negative & Negative & Negative & $\# 2$ & Positive & Positive & Positive \\
\hline & $\# 3$ & Negative & Negative & Negative & $\# 3$ & Positive & Positive & Positive \\
\hline & $\# 4$ & Negative & Negative & Negative & $\# 4$ & Positive & Positive & Positive \\
\hline & $\# 5$ & Negative & Negative & Negative & $\# 5$ & Positive & Positive & Positive \\
\hline \multirow{5}{*}{3} & $\# 1$ & Negative & Negative & Negative & $\# 1$ & Positive & Positive & Positive \\
\hline & $\# 2$ & Negative & Negative & Negative & $\# 2$ & Positive & Positive & Positive \\
\hline & $\# 3$ & Negative & Negative & Negative & $\# 3$ & Positive & Positive & Positive \\
\hline & $\# 4$ & Negative & Negative & Negative & $\# 4$ & Positive & Positive & Positive \\
\hline & $\# 5$ & Negative & Negative & Negative & $\# 5$ & Positive & Positive & Positive \\
\hline
\end{tabular}

Data from Brooks, B. et al. ${ }^{2}$

head. When tested up to 20 HPV cycles, no visible degradation was observed. After $30 \mathrm{HPV}$ cycles, the elastic material in the FFR straps were susceptible to fragmentation upon stretching, which indicates a potential FFR failure if the straps were to break. As such, a recommendation is made to identify alternative materials for the FFR strap that are more resistant to HPV decontamination. Moreover, the aerosol collection efficiency was found to be unaffected over the course of the 50 cycles of HPV decontamination, as seen in Table 2. However, an important note is that further testing is recommended with different brands of FFR to ensure adherence to this important finding. ${ }^{2}$

Phase III confirmed that decontamination of the N95 FFR can still be achieved despite undergoing 50 cycles of HPV decontamination, with complete inactivation of the organism, as seen in Table 3 . This demonstrates the utility of maintained cycle effectiveness in decontamination. ${ }^{2}$

As such, this pilot-study demonstrated the efficacy of decontaminating N95 FFRs and indicates that the use of HPV decontamination will adequately decontaminate FFRs, while maintaining the integrity of the respirator until up to 30 cycles (upon which the straps may need to be replaced), and can be effectively decontaminated for at least 50 cycles, maximum number tested (upper limit unknown). The resultant finding is limited since the investigators aimed at exploring the efficacy of using HPV as part of a plausible decontamination protocol for respirators but tested only one type of N95 FFR, Model 1860 (3M, St. Paul, MN). The study was also conducted by a not-for-profit applied science and technology development company Battelle, which endorses the use of the commercially available Bioquell Clarus $\mathrm{C}$ system. ${ }^{2}$

\section{Ultraviolet Germicidal IRRAdiation}

Vescusi et al attempted to evaluate five separate decontamination methods-Ultraviolet Germicidal Irradiation (UVGI), ethylene oxide (EtO), microwave oven irradiation, bleach, Hydrogen Peroxide Vapor 
Table 2. Summary of Bioaerosol Collection Efficiency Results (HPV FFRs)

\begin{tabular}{|l|c|c|c|c|c|c|}
\hline \multirow{2}{*}{ HPV Cycles } & \multicolumn{7}{|c|}{ Collection Efficiency (\%) } \\
\cline { 2 - 7 } & $\mathbf{\# 1}$ & $\mathbf{\# 2}$ & $\mathbf{\# 3}$ & $\mathbf{\# 4}$ & $\mathbf{\# 5}$ & Average \\
\hline 10 & 99.5 & $>99.9$ & 99.7 & 99.9 & 99.6 & $>99.5$ \\
\hline 20 & $>99.9$ & $>99.9$ & $>99.9$ & $>99.9$ & $>99.9$ & $>99.9$ \\
\hline 30 & $>99.9$ & $>99.9$ & $>99.9$ & $>99.9$ & $>99.9$ & $>99.9$ \\
\hline 40 & $>99.9$ & $>99.9$ & $>99.9$ & $>99.9$ & $>99.9$ & $>99.9$ \\
\hline 50 & $>99.9$ & $>99.9$ & $>99.9$ & $>99.9$ & $>99.9$ & $>99.9$ \\
\hline
\end{tabular}

Data from Brooks, B. et al. ${ }^{2}$

(HPV) and a control group on nine different respirators approved by the National Institute for Occupational Safety and Health (NIOSH). These respirators were analyzed for any changes in physical appearance, odor, and performance (aerosol filtration and airflow resistance). Microwave irradiation melted two of the sample respirators. It was found that the respirators treated with the other methods were decontaminated and had expected levels of performance; however, it was noted that the respirators treated with bleach still had a bleach odor even after drying overnight. The results showed that UVGI, EtO, and HPV were promising decontaminating methods. The investigators indicated, however,

Table 3. Summary of Results from the Multiple Cycle Decontamination Efficiency Testing

\begin{tabular}{|l|l|l|l|}
\hline \multirow{2}{*}{ FFR } & \multicolumn{2}{|c|}{$\begin{array}{c}\text { Growth/No Growth } \\
\text { Observations }\end{array}$} & \multirow{2}{*}{$\begin{array}{l}\text { Streak } \\
\text { Plate }\end{array}$} \\
\cline { 2 - 3 } Day 4 & Day 7 & Negative \\
\hline HPV \#1 & Negative & Negative & Negative \\
\hline HPV \#2 & Negative & Negative & Negative \\
\hline HPV \#3 & Negative & Negative & Negative \\
\hline HPV \#4 & Negative & Negative & Negative \\
\hline HPV \#5 & Negative & Negative & Positive \\
\hline Control \#1 & Positive & Positive & Positive \\
\hline Control \#2 & Positive & Positive & Pose \\
\hline Control \#3 & Positive & Positive & Positive \\
\hline Control \#4 & Positive & Positive & Positive \\
\hline Control \#5 & Positive & Positive & Positive \\
\hline
\end{tabular}

Data from Brooks, B. et al. ${ }^{2}$ that further research was required before a specific decontamination method could be recommended. ${ }^{3}$

Protocol methods included a control group that received no treatment. The UVGI group was exposed to 40-W UV light, with an intensity range from 0.18 to $0.20 \mathrm{~mW} / \mathrm{cm}^{2}$, for 15 minutes on each side of the respirator (176-181 $\mathrm{mJ} / \mathrm{cm}^{2}$ ). The EtO decontamination utilized a Steri-Vac $5 \mathrm{XL}$ sterilizer with a single warm cycle at $55^{\circ} \mathrm{C}$ and $725 \mathrm{mg} / \mathrm{L} 100 \%$ EtO Gas; a one-hour EtO exposure was followed by 4 hours of aeration. Hydrogen Peroxide Vapor (HPV) decontamination used the STERRAD ${ }^{\circledR} 100 \mathrm{~S} \mathrm{H}_{2} \mathrm{O}_{2}$ Gas Plasma Sterilizer for a single 55-minute cycle. A $2450 \mathrm{MHz}$, Sharp Model R-305KS was used for microwave oven irradiation. Respirators were exposed for 1 minute on each side at the maximum power setting of 10 , $1100 \mathrm{~W}, 750 \mathrm{~W} / \mathrm{Ft}^{3}$. Due to microwave irradiation causing some of the FFRs to melt, the researchers tested dry oven treatments at 100,110 , and $120^{\circ} \mathrm{C}$. $^{3}$

This study found that UVGI and EtO treatment did not affect the performance or physical appearance of the respirators, which were also effectively decontaminated. Hydrogen Peroxide Vapor was effective at temperatures ranging from $4-80^{\circ} \mathrm{C}$, with concentrations varying $0.5-<10 \mathrm{mg} / \mathrm{L}$. Similarly, a single cycle of HPV did not have a significant effect on respirator performance or appearance. While bleach did not affect the appearance or performance of the treated respirators, the residual odor of bleach remaining afterward was concerning. The potential health risks from even low concentrations of this chemical led the researchers to discourage the usage of bleach for decontamination. Microwave irradiation treatment caused melting, 
and dry oven heat at 100,110 and $120^{\circ} \mathrm{C}$ also melted some of the respirators. Researchers stated that possible modifications to microwave irradiation and dry oven techniques may lead to limited success. Final results indicated that UVGI, EtO and HPV techniques appear to be the most promising decontamination protocols among the methods evaluated. ${ }^{3}$

A major limitation of this study is that no testing with subsequent cycles of decontamination involving any of the decontaminating methods was carried out. In addition, the resultant findings can only be applied to the nine specific respirators used in these trials. Furthermore, cost-effectiveness of the testing methods was not evaluated in this investigation. This study was conducted and funded by the National Institute for Occupational Safety and Health (NIOSH). ${ }^{3}$

A protocol was recently developed by the Nebraska Medicine group utilizing UVGI to decontaminate used N95 respirators in order to be safely reused by healthcare workers. As demonstrated in Figure 1, design method utilized two UVGI towers on opposite sides of the room placed eight feet apart, with a line for the N95 respirators to be hung on half the distance in between with a UV light sensor present. The $13 \mathrm{ft}$ lines were capable of holding 30 respirators at any one time. This process exposed the respirators to $60 \mathrm{~mJ} / \mathrm{cm}^{2}$ of UGVI. The researchers stated that only $2-5 \mathrm{~mJ} / \mathrm{cm}^{2}$ was required to inactivate single stranded RNA viruses and that the decontamination process duration of only 5-6 minutes was needed to reach the required UVGI exposure level. A major advantage to this protocol was the number and rate at which a sizable number FFRs can be decontaminated at once. ${ }^{4}$

A study by Lindsey et al indicated UV levels as low as $120 \mathrm{~mJ} / \mathrm{cm}^{2}$ affected the integrity of the respirators and that higher levels caused even more damage. ${ }^{5}$ Figure 2 demonstrates the varying effectiveness of different UV wavelengths. The protocol from Nebraska Medicine stated that the "levels of UVGI needed to inoculate human respiratory viruses are well below the level of irradiation that adversely affects the fit and filtration characteristics of the N95 FFRs." ${ }^{4}$ This finding does align with the results found in the study published by $\mathrm{NIOSH} .{ }^{3}$ This is further supported by the findings of Tseng et al researchers showing that $90 \%$ viral reduction could be achieved with UV exposure of $1.32-3.20 \mathrm{~mJ} / \mathrm{cm}^{2}$ for single-stranded RNA and 2.5 to $4.47 \mathrm{~mJ} / \mathrm{cm}^{2}$ for double-stranded DNA. ${ }^{6}$ Therefore, the $60 \mathrm{~mJ} / \mathrm{cm}^{2}$ of UGVI utilized in the protocol developed by Nebraska Medicine should be sufficient for decontamination and possibly not damage the N95 FFRs. However, thus far, no study has analyzed the effects of repeated UVGI cycles at a certain intensity on any respirators. ${ }^{4}$

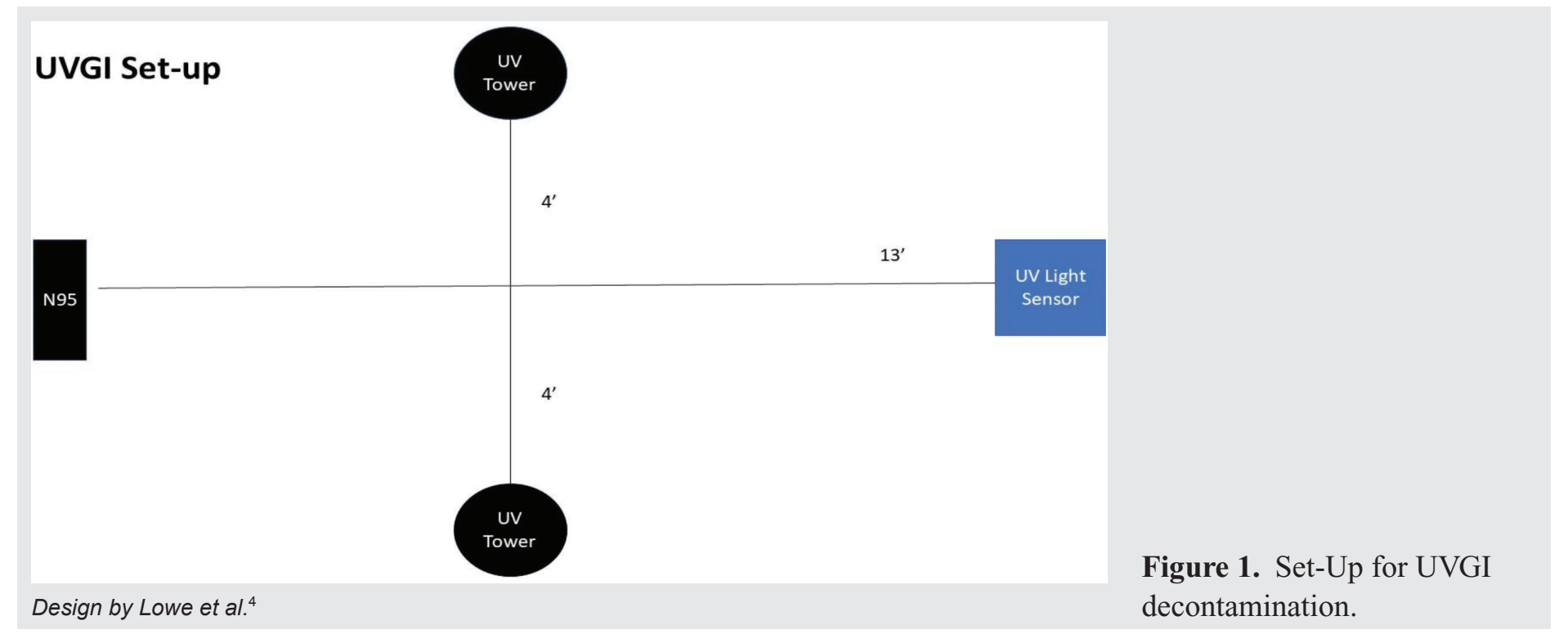

The Southwest Respiratory and Critical Care Chronicles 2020;8(34):27-39 


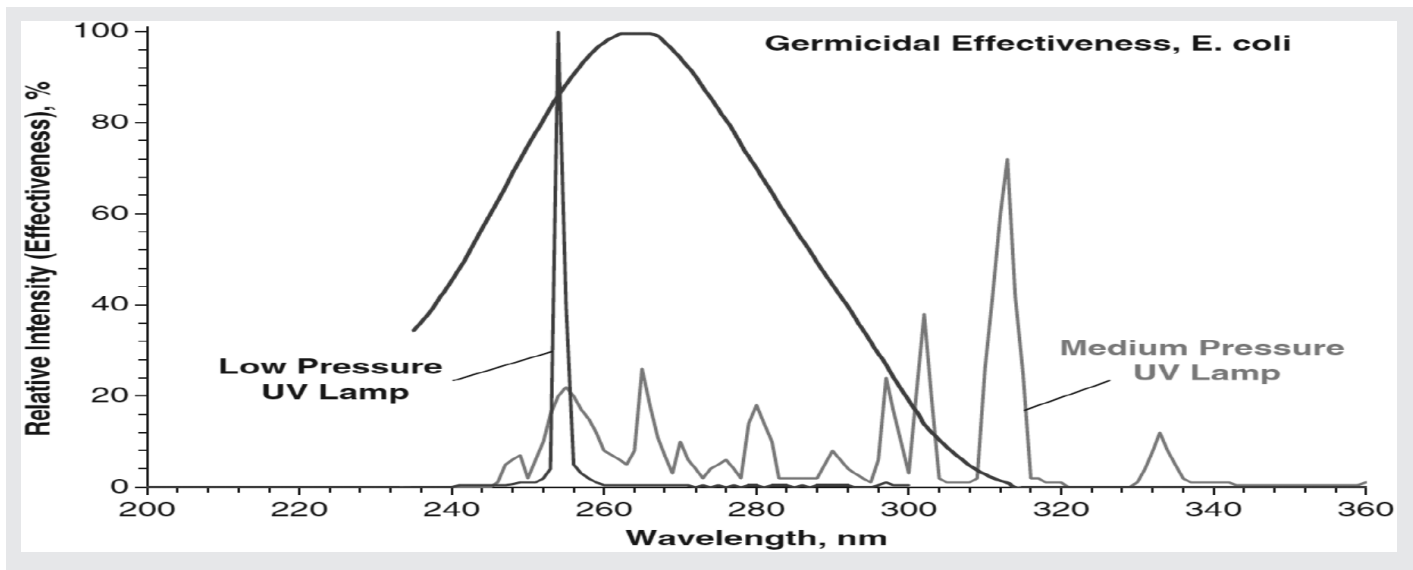

(Graphic from Creative Commons)
Figure 2. Various UV wavelengths' effectiveness.

\section{Decontamination Method Comparisons}

On behalf of 4C Air Inc., Stanford Anesthesiology has published rudimentary data on the efficacy of various methods of decontamination of N95 respirators. Table 4 demonstrates their findings. Data supplied courtesy of:

Professor Yi Cui | Materials Science and Engineering, Stanford University and Professor Steven Chu I Physics and Molecular \& Cellular Physiology, Stanford University on behalf of 4C Air Incorporated. ${ }^{7}$

The conclusions are as follows: alcohol and chlorine-based decontamination or disinfection methods are not recommended for use on N95 respirators since these chemicals will remove the static charge in the microfibers, thus reducing the integrity of the system and diminishing the respirator's filtration efficiency. Moreover, chlorine retains gas after decontamination and could result in harmful fumes being inhaled. The efficacy of other methods has been tested and are confirmed to be better solutions to the shortage of PPE. ${ }^{7}$

These preliminary data indicate that utility possibly exists in the development and fine-tuning of a protocol to disinfect and reuse PPE during the supplychain shortage in the current COVID-19 pandemic. Moreover, it is important to note that the various decontamination methods demonstrate differences in filtration efficiency and pressure drop changes, and as such should be examined carefully prior to undergoing decontaminating measures. ${ }^{7}$

\section{Table 4. Evaluation of Decontamination Techniques}

\begin{tabular}{|c|c|c|c|c|c|}
\hline \multirow[b]{2}{*}{ Samples } & \multicolumn{2}{|c|}{ Melt-blown Fiber Filtration Media } & \multicolumn{2}{|c|}{ Static-charged Cotton } & \multirow{2}{*}{$\begin{array}{c}\text { E. coli } \\
\text { Disinfection } \\
\text { Efficiency }\end{array}$} \\
\hline & $\begin{array}{c}\text { Filtration } \\
\text { Efficiency (\%) }\end{array}$ & $\begin{array}{c}\text { Pressure Drop } \\
\text { (Pa) }\end{array}$ & $\begin{array}{c}\text { Filtration } \\
\text { Efficiency }(\%)\end{array}$ & $\begin{array}{l}\text { Pressure } \\
\text { Drop (Pa) }\end{array}$ & \\
\hline $70^{\circ} \mathrm{C}$ hot air in oven, $30 \mathrm{~min}$ & 96.60 & 8.00 & 70.16 & 4.67 & $>99 \%$ \\
\hline UV light, $30 \mathrm{~min}$ & 95.50 & 7.00 & 77.72 & 6.00 & $>99 \%$ \\
\hline 75\% alcohol, soaking and drying & 56.33 & 7.67 & 29.24 & 5.33 & $>99 \%$ \\
\hline Chlorine-based disinfection, $5 \mathrm{~min}$ & 73.11 & 9.00 & 57.33 & 7.00 & $>99 \%$ \\
\hline $\begin{array}{l}\text { Hot water vapor from boiling } \\
\text { water, } 10 \mathrm{~min}\end{array}$ & 94.74 & 8.00 & 77.65 & 7.00 & $>99 \%$ \\
\hline Initial samples before treatment & 96.76 & 8.33 & 78.01 & 5.33 & \\
\hline
\end{tabular}

Data from Stanford Medicine et al. ${ }^{7}$ 


\section{EXTENDED UsE}

The Centers for Disease Control (CDC) has established protocols for pandemic planning. These recommended practices are for healthcare workers facing dwindling supplies of N95 respirators during an influenza pandemic or wide-spread outbreaks of other infectious respiratory illnesses. Among the practices that the CDC indicates in similar situations (such as the COVID-19 pandemic) is a recommendation to "implement practices allowing extended use and/or limited reuse of $\mathrm{N} 95$ respirators, when acceptable...."

Moreover, extended use and reuse were defined as the following:

Extended use refers to the continuous wear of an N95 respirator for the treatment of several patients who are suffering from the same infectious disease and therefore cannot infect one another. Instead, this practice allows the wearer to minimize risk from personal transmission of the pathogen by minimizing the removal of the respirator, which may preserve the functionality and fit over time. Extended use is a recommendation of the CDC for the conservation of respirators in a setting of PPE shortage. ${ }^{8}$

Reuse refers to the practice of using the same N95 respirator for multiple encounters with patients, while taking it off and putting it back on between patients. The practice of reusage is recommended to be employed for only a limited number of encounters. Limited reuse has been recommended and widely practiced as an option for conserving respirators during previous respiratory pathogen outbreaks and pandemics. $^{8}$

Such definitions allow others to better understand the efficacy of extended usage and reuse of respirators, and the CDC summarizes this protocol with the following recommendation:

"Extended use is preferred over reuse because it is expected to involve less touching of the respirator and therefore reduced risk of contact transmission...A key consideration for safe extended use is that the respirator must maintain its fit and function."
As such, CDC recommendations allow for the extended usage and reuse of respirators by healthcare workers in the setting of respirator supply shortages. Moreover, it is key for hospital and healthcare system administrators to institute these conservation strategies on a system-wide level along with careful consideration to implementation. The priority of respirator utilization should be evaluated at every level, and efforts should be made on a system-wide basis to aid in the conservation of PPE. Worse case scenarios occur when these attempts at preservation culminate in disorganization and confusion, which can prove to be disastrous in a pandemic setting. Given the role of the CDC, no plausible conflict of interest or bias is expected..$^{9,10}$

\section{Homemade Masks}

In the event that proper PPE such as N95 FFRs and standard surgical masks become unavailable, some have turned to household items as alternatives. Researchers at Cambridge University investigated the efficacy of homemade masks for protection. An important distinction is the difference between a mask and a respirator; surgical masks can protect against droplets but they are not as effective as respirators at protecting against aerosols. The purpose of this research is not to substantiate claims that household items are adequate replacements for medical masks, but rather to determine which type of material would perform best for substitution if proper PPE becomes depleted. Davies et al tested different household items being used as makeshift masks including: 100\% cotton T-shirt, scarf, tea towel, pillowcase, antimicrobial pillowcase, surgical mask, vacuum cleaner bag, cotton mix, linen, and silk. Results of the efficacy of each material is demonstrated in Table 5. The number of microorganisms isolated from coughs of healthy volunteers were compared while wearing homemade masks, surgical masks, and no masks. These researchers found that homemade masks should be considered a last resort for prevention but would fare better than no protection at all. ${ }^{11}$

For the purpose of this study, surgical masks (pictured in Figure 3) were used as the control for comparison to homemade masks. A Henderson apparatus 
Table 5. Mean Performance of Household Materials

\begin{tabular}{|l|c|c|}
\hline Material & $\begin{array}{c}\text { Mean \% Filtration } \\
\text { Efficiency }\end{array}$ & $\begin{array}{c}\text { Mean Pressure Drop } \\
\text { Across Fabric }\end{array}$ \\
\hline 100\% Cotton T-shirt & 60.2 & 4.29 \\
\hline Scarf & 55.6 & 4.36 \\
\hline Tea Towel & 77.9 & 7.23 \\
\hline Pillowcase & 59.2 & 3.88 \\
\hline Antimicrobial Pillowcase & 67.3 & 6.11 \\
\hline Vacuum Cleaner Bag & 90.2 & 10.18 \\
\hline Cotton Mix & 72.4 & 6.18 \\
\hline Linen & 60.8 & 4.5 \\
\hline Silk & 56.2 & 4.57 \\
\hline Surgical Mask & 92.9 & 4.57 \\
\hline
\end{tabular}

Data from Davies et al. ${ }^{11}$

was used to test the filtration efficiency; this instrument delivered several aerosols across the variety of materials at $30 \mathrm{~L} /$ minute. This rate of spraying is roughly 3 times the normal ventilation of a person at rest. The pressure drop across the different fabrics was also measured using a manometer. Two microorganisms, bacteriophage MS2 and Bacillus atrophaeus, were used to test filtration efficacy because they are of similar size to the influenza virus. ${ }^{11}$

It is worth noting that all tests were done with fresh unused material, thus that same fabric if worn for several hours may yield very different results. ${ }^{11}$ Another point of concern is that if these masks become

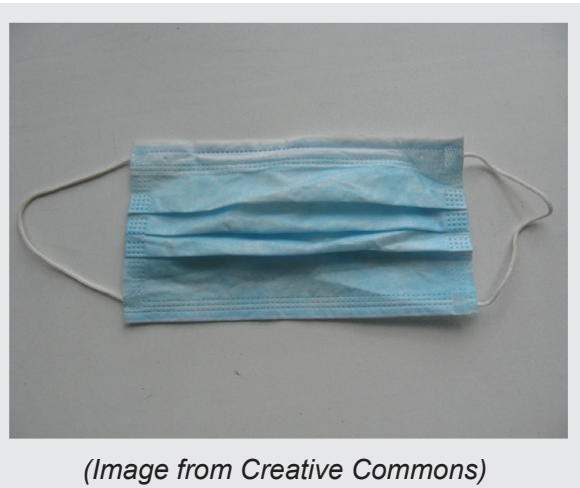

Figure 3. Surgical Mask. potentially contaminated, they may propagate the spread of disease rather than prevent it. In the event that a wearer believes the homemade mask may have been contaminated, it would be best to follow military protocol for clothing decontamination. As soon as possible, the wearer should remove the mask, place it in a sealed plastic bag, and wash using $100^{\circ} \mathrm{F}$ water with either 1 -pound soap/10 gallons of water or $5 \%$ sodium-carbonate solution for one hour. As always, proper hand hygiene should also be practiced as well. ${ }^{12}$

The above study also found that any type of manufactured medical mask will reduce the spread of possible microorganisms or viral particles and would provide some protection against droplets containing potential pathogens. However, homemade masks offer little protection to the wearer from infection transmitted through aerosols. Table 6 demonstrates how much bacteria was transmitted by a surgical mask wearer, DIY mask user and no mask. The researchers stated that a homemade mask will not provide much benefit if it is not used in conjunction with other preventive measures/protocols such as proper hand hygiene and isolation of infected matter. While the vacuum cleaner bag had the highest filtration efficiency out of all tested household materials, it was also the most difficult through which to breathe. This feature caused this mask to not have a good fit and seal when worn. Doubling the material was also tested to 
Table 6. Total Colony-Forming Units Isolated by Particle Size

\begin{tabular}{|l|c|c|c|}
\hline Particle Diameter, $\boldsymbol{\mu M}$ & No Mask & *Homemade Mask & Surgical Mask \\
\hline$>7$ & 9 & 3 & 5 \\
\hline $4.7-7$ & 18 & 7 & 7 \\
\hline $3.3-4.7$ & 5 & 4 & 4 \\
\hline $2.1-3.3$ & 47 & 7 & 5 \\
\hline $1.1-2.1$ & 100 & 16 & 6 \\
\hline $0.65-1.1$ & 21 & 6 & 30 \\
\hline Total & 200 & 43 & \\
\hline
\end{tabular}

*Homemade masks were made from $100 \%$ cotton T-shirts

Data from Davies et al. ${ }^{11}$

determine if it would increase the filtration efficiency, but it was found that the filtering mechanism did not significantly increase in effectiveness, but the effort of breathing did rise in difficulty. ${ }^{11}$ Another study also showed that even using up to 5 layers of surgical masks did not compare to the filtration capacity of a N95 FFR. ${ }^{13}$ On the other hand, it was found that having a good fit and sensation of comfort are important features of a homemade mask. The stretchy quality of cotton T-shirts made it the more optimal choice for a non-medical mask. In the end, medically approved proper PPE offers much more protection than a homemade mask, but a homemade mask is better than no mask at all. ${ }^{11}$

\section{Reusable Gowns}

Finally, we reviewed a study determining the efficacy of laundering reusable surgical gown fabrics, which is an essential component of PPE that acts as a physical barrier to the transmission of infectious droplets and aerosols produced by body fluids. It has been demonstrated that following laundering, the type of fabric used in a surgical gown can dramatically influence the physical properties of the gown itself. By investigating the repellency and pore size of various fabric types from which gowns are manufactured, it was shown that some materials will exhibit loss of inherent protective feature upon laundering, while others were less affected. ${ }^{14}$

The researchers at the University of Georgia compared five different reusable gowns made up of various fiber types, listed in the tables below. All of the gowns were then subjected to the same, but separate washing cycles and data were collected at zero laundering, 25 launderings, and 50 launderings. ${ }^{14}$ These garments were all gowns intended to be washed and reused and not single-use gowns, the different fiber types are demonstrated in Table 7. Single-use polypropylene gowns have become the dominant choice to wear while performing surgeries because they offer better protection than reusable gowns. ${ }^{15}$

Table 10 shows fabric $A$ is the only type of material that demonstrated no transmission of bacteria after laundering. This finding was significant since this fabric retained the greatest thickness and highest degree of water repellency as demonstrated in Tables 8 and 9,

Table 7. Reusable Gowns Descriptions

\begin{tabular}{|l|l|l|}
\hline Code & Fiber & Construction \\
\hline $\mathrm{A}^{*}$ & $99 \%$ polyester $1 \%$ carbon & Plain $^{\dagger}$ \\
\hline $\mathrm{B}$ & $100 \%$ polyester & Plain \\
\hline $\mathrm{C}$ & $50 \%$ cotton$/ 50 \%$ polyester & Plain \\
\hline $\mathrm{D}$ & Face $100 \%$ polyester & Composite \\
& Middle Gore-Tex & Plain \\
& Back $100 \%$ polyester & Membrane Jersey \\
& $100 \%$ polyester & Plain \\
\hline$E^{*}$ & \multicolumn{2}{|l}{} \\
\hline
\end{tabular}

${ }^{*}$ Fabric reinforced.

†Woven.

${ }^{\ddagger}$ Knit.

Data from Leonas et al. ${ }^{14}$ 
Table 8. Mean Fabric Physical Characteristics

\begin{tabular}{|l|c|c|c|l|}
\hline Fabric & 0 Launderings & 25 Launderings & 50 Launderings & \\
\hline \multirow{2}{*}{$\mathrm{A}$} & 178.2 & 185.8 & 188.5 & Weight $\mathrm{g} / \mathrm{m}^{2}$ \\
\cline { 2 - 5 } & 230 & 152.2 & 152.4 & Thickness $\mu \mathrm{m}$ \\
\hline \multirow{2}{*}{$\mathrm{B}$} & 5.3 & 136.0 & 137.2 & Weight $\mathrm{g} / \mathrm{m}^{2}$ \\
\cline { 2 - 5 } & 23 & 224.5 & 169.9 & Thickness $\mu \mathrm{m}$ \\
\hline \multirow{2}{*}{$\mathrm{C}$} & 98.5 & 100.9 & 103.3 & Weight $\mathrm{g} / \mathrm{m}^{2}$ \\
\cline { 2 - 5 } & 123 & 142.7 & 169.9 & Thickness $\mu \mathrm{m}$ \\
\hline \multirow{2}{*}{$\mathrm{D}$} & 179.2 & 195.3 & 200.2 & Weight $\mathrm{g} / \mathrm{m}^{2}$ \\
\cline { 2 - 5 } & 395 & 440.5 & 457.2 & Thickness $\mu \mathrm{m}$ \\
\hline \multirow{2}{*}{$\mathrm{E}$} & 169.8 & 175.2 & 177.4 & Weight $\mathrm{g} / \mathrm{m}^{2}$ \\
\cline { 2 - 5 } & 183 & 121.9 & 115.3 & Thickness $\mu \mathrm{m}$ \\
\hline
\end{tabular}

Data from Leonas et al..$^{14}$

Table 9. Mean Fabric Repellency Characteristics

\begin{tabular}{|l|c|c|c|l|}
\hline Fabric & 0 Launderings & 25 Launderings & 50 Launderings & \\
\hline \multirow{2}{*}{ A } & 100 & 86 & 62 & Water Repellency Rating \\
\cline { 2 - 5 } & 5.8 & 4.8 & 0.2 & Oil Repellency Rating \\
\hline \multirow{2}{*}{ B } & 100 & 74 & 0 & Water Repellency Rating \\
\cline { 2 - 5 } & 6 & 2.2 & 0 & Oil Repellency Rating \\
\hline \multirow{2}{*}{ C } & 100 & 76 & 0 & Water Repellency Rating \\
\cline { 2 - 5 } & 4.6 & 0.6 & 0 & Oil Repellency Rating \\
\hline \multirow{2}{*}{ D } & 0 & 50 & 50 & Water Repellency Rating \\
\cline { 2 - 5 } & 0 & 0 & 0 & Oil Repellency Rating \\
\hline \multirow{2}{*}{ E } & 100 & 90 & 0 & Water Repellency Rating \\
\cline { 2 - 5 } & 2.2 & 0 & 0 & Oil Repellency Rating \\
\hline
\end{tabular}

Data from Leonas et al. ${ }^{14}$

Table 10. Average Bacterial Transmission (CFUs)

\begin{tabular}{|l|c|c|c|}
\hline Fabric & 0 Launderings & 25 Launderings & 50 Launderings \\
\hline A & 0 & 0 & 0 \\
\hline B & 1.5 & 87.3 & 0 \\
\hline C & 0 & 76.8 & 77 \\
\hline D & 217 & 45.5 & 34.6 \\
\hline E & 0.3 & .7 & 0 \\
\hline
\end{tabular}

Data from Leonas et al. ${ }^{14}$ 
respectively. ${ }^{14}$ Two other fabrics demonstrated little to no change in transmission of bacteria after laundering, and these fabric types were found to be reinforced with two layers of material as well. As such, for conservation of PPE, material thickness and repellency are two significant factors that should be considered when deciding to reuse laundered gowns. ${ }^{14}$ It is also important to note that the majority of surgical gowns and hospital gowns in general are produced as single-use, and as such are not compatible with laundering. These results do show that a reusable gown constructed of $99 \%$ polyester and $1 \%$ carbon offers the most protection from transmitting bacteria. This research involved launderable gowns that are intended for reuse, and these findings provide guidance for healthcare workers looking to improvise by making effective gowns from available materials. ${ }^{14}$ Currently, there is paucity of data about decontaminating reusable gowns, so one must again default to the military process for decontaminating cloth material. As soon as possible after potential contamination, the gown should be placed in a sealed plastic bag and washed using $100^{\circ} \mathrm{F}$ water with either 1-pound soap/10 gallons of water or $5 \%$ sodium-carbonate solution for one hour. ${ }^{12}$

The investigation was conducted and funded by the University of Georgia. This study was limited to the five reusable gowns tested. There was no declared conflict of interest or bias.

\section{Discussion}

This literature review presents clinically relevant findings that should be considered when making an institutional decision regarding a hospital or healthcare system's response to a shortage or absence of N95 respirators and other personal protective equipment (PPE). In the pursuit of safe and feasible alternatives to standard PPE in the setting of an infectious respiratory illness, such as the current COVID-19 pandemic, available data demonstrate the following:

The use of hydrogen peroxide vapor (HPV) generated by the Bioquell decontamination system will: 1) adequately decontaminate FFRs, 2) maintain the integrity of the respirator until up to 30 cycles (upon which the straps may need to be replaced), and 3) can be effectively decontaminated for 50 cycles (upper limit unknown, maximal number of tested cycles $=50$ ). These findings are supported by data that indicate: 1) HPV cycles decontaminate FFRs and will test negative for organisms after one cycle, 2) the integrity of attached straps will begin to diminish around cycle 30 but the collection efficiency is maintained through numerous cycles, and 3 ) after 50 cycles of decontamination the FFRs will still test negative for organisms and remain safe for usage. This study is important for hospital and healthcare system administrators who wish to implement safe and effective standardized protocols for the decontamination/disinfection of any limited supply of PPE. It is important to note, however, that the efficacy of this protocol was demonstrated for just one specific brand, the N95 respirator, Model 1860 (3M, St. Paul, MN). As such, organizational system personnel who wish to use other respirator models should test this protocol prior to instituting HPV decontamination as standard practice to ensure safety of healthcare workers. ${ }^{2}$

The protocol for decontaminating N95 FFRs using UVGI developed by Nebraska Medicine is based on scientific evidence showing that UVGI can effectively decontaminate used respirators without hindering performance or compromising the integrity of the FFR. However, there has yet to be a study conducted on the effect of repeated UVGI cycles. Until such evidence is available, it is not certain that repeated UVGI cycles will not damage the FFRs. After further research, UVGI may become a useful method for decontaminating PPE. ${ }^{4}$

The CDC has outlined specific protocols for pandemic planning. These recommended practices are set in place for healthcare workers with a shortage of N95 respirators during an influenza pandemic or wide-spread outbreaks of other infectious respiratory illnesses. As such, data may be extrapolated and the CDC's recommendations may be applied to the COVID-19 pandemic for instructing hospital plus other healthcare system personnel to institute the following practices at the administrators' discretion: 
Extended use of PPE is permissible and is defined as wearing the same N95 respirator for repeated close contact encounters with several patients, without removing the respirator between patient encounters. Prolonged respirator usage may be implemented when multiple patients are infected with the same respiratory pathogen and are placed together/isolated in dedicated waiting rooms or hospital wards. In these circumstances, extended use of respirators minimizes the opportunities for healthcare workers to infect themselves by minimizing removal and re-wearing of the same respirator. As such, prolonged usage may be implemented, emphasizing that workers should not fidget with the respirators while worn to minimize altering the shape/fit causing respirator malfunction and therein infection. ${ }^{8}$

Alcohol and chlorine-based disinfection methods should not be used on N95 respirators, as they damage the physical integrity of the respirator by removing the static charge in the microfibers. Moreover, chlorine-based disinfection may result in residual chlorine that retains gas after decontamination. ${ }^{7}$

It was determined that homemade masks do indeed help prevent the spread of disease, but do not offer much protection against infectious aerosols. Homemade masks may be better suited to be worn by those who suspect they may be infected to prevent spreading disease to others, rather than protecting the healthy from contracting an illness. These scientists concluded that wearing a homemade mask is better than no mask but does not offer the same level of protection as a medical mask. They recommend that homemade masks be worn only as a last resort, and that these be used in conjunction with other preventative infection control measures. ${ }^{11}$

In addition to homemade alternatives for masks, healthcare workers may need to reuse surgical and hospital gowns. As such, the laundering of reusable garments must be approached with an understanding of the properties of fabrics that make the gown an effective PPE. This review discusses both repellency and thickness of the fabric materials to be indicators of the protection against pathogenic transmission through the gown after laundering. The protective quality of a fabric consisting of $99 \%$ polyester and $1 \%$ carbon was not affected by up to 50 launderings thus is deemed as ideal for reusage. ${ }^{14}$

The limitations of this current review stem from the nature of the virus at hand, which is unique. Previous investigations have demonstrated the efficacy of a number of different decontamination methods. However, these investigations were often preliminary and specific in nature. The ability to extrapolate definitive conclusions from such studies must be approached carefully to ensure the safety of healthcare workers who use decontaminated and reused respirators, extended used respirators, or homemade/DIY masks. However, this article provides hospital personnel and healthcare system administrators with information to better understand the available literature on this topic and to make informed decisions regarding the safety of their staff. Moreover, limitations exist on precise understanding of the intricacies of transmission modes regarding the coronavirus, SARS CoV-2, thus further confounding an evolving big picture.

\section{Conclusions}

In conducting this comprehensive review, available data were analyzed to help establish sound guiding principles for hospitals and healthcare system administrators to make informed decisions about the PPE shortage during the COVID-19 pandemic. As such, the following recommendations can be made, while emphasizing the need for a coordinated and well thought out institutional response:

With regard to decontaminating used N95 respirators, current evidence supports the use of HPV as the long-term solution. Much research exists indicating that this method effectively decontaminates the FFRs with little risk of damaging the integrity or compromising the performance of the respirator for up to 50 cycles. $^{2}$

The CDC emphasizes the utility in maintaining the fit of respirators, and this can be achieved by extending the length of usage as opposed to reusing the FFR, which would involve removing the respirator and potentially compromising the tight seal fit. These guidelines are likely to be effective in treating a group 
of COVID-19 patients, as these patients will be isolated with the same virus in one enclosed area. ${ }^{7}$

Current evidence also shows that homemade masks may have a minor role in preventing the spread of infectious disease, thus should only be utilized as a last resort and in combination with other preventative infection control measures. ${ }^{11}$ Lastly, when selecting a reusable gown, current research supports using fabric material consisting of $99 \%$ polyester and $1 \%$ carbon for the best chance of protection. ${ }^{14}$

Article citation: Kharbat A, Mizer A, Zumwalt M. Decontamination methods of personal protective equipment for repeated utilization in medical/surgical settings. The Southwest Respiratory and Critical Care Chronicles 2020;8(34):27-39

From: The Department of Orthopedics (MZ) and the School of Medicine (AK,AM), Texas Tech University Health Sciences Center, Lubbock, Texas

Submitted: $4 / 2 / 2020$

Accepted: 4/6/2020

Reviewer: Kenneth Nugent MD

Conflicts of interest: none

This work is licensed under a Creative Commons

Attribution-ShareAlike 4.0 International License.

\section{REFERENCES}

1. Roberge RJ. Face shields for infection control: A review. J Occup Environ Hyg 2016;13(4):235-242. doi:10.1080/1545 9624.2015.1095302

2. Brooks B. Final report for Bioquell HPV decontamination for reuse of N95 respirators. Silver Spring, MD: FDA Office of Counterterrorism and Emerging Threats; July 22, 2016. 46 p. Report No.: 3245

3. Viscusi DJ, Bergman MS, Eimer BC, et al. Evaluation of five decontamination methods for filtering facepiece respirators. Ann Occup Hyg 2009;53(8):815-827. doi:10.1093/annhyg/ mep070

4. Lowe JJ, Paladino KD, Farke JD, et al. N95 filtering facepiece respirator ultraviolet germicidal irradiation (UGVI) process for decontamination and reuse. Nebraska Medicine March 212020.

5. Lindsley WG, Martin Jr SB, Thewlis R E, et al. Ultraviolet germicidal irradiation (UVGI) on N95 respirator filtration performance and structural integrity. Journal of Occupational and Environmental Hygiene 2015;12(8):509-517, DOI: $10.1080 / 15459624.2015 .1018518$

6. Tseng CC, Li CS. Inactivation of viruses on surfaces by ultraviolet germicidal irradiation. 2007. Journal of Occupational and Environmental Hygiene, 4:6, DOI: 10.1080/ 15459620701329012

7. Anesthesia Informatics and Media Lab. Addressing COVID19 face mask shortages [V1.1]. Stanford Medicine 2020 Mar 22. Unpublished Manuscript.

8. CDC-Recommended guidance for extended use and limited reuse of N95 filtering facepiece respirators in healthcare settings-NIOSH Workplace Safety and Health Topic Centers for Disease Control and Prevention, 28 Mar. 2018. Unpublished Manuscript. Available from: www.cdc.gov/niosh/topics/ hcwcontrols/recommendedguidanceextuse.html

9. SARS. Centers for Disease Control and Prevention, 3 May 2005. Unpublished Manuscript. Available from: www.cdc. gov/sars/clinical/respirators.html.

10. Institute of Medicine. Reusability of facemasks during an influenza pandemic: Facing the flu. Washington, DC: The National Academies Press. 2006. https://doi.org/10.17226/ 11637

11. Davies A, Thompson K-A, Giri K, et al. Testing the efficacy of homemade masks: Would they protect in an influenza pandemic? Disaster Medicine and Public Health Preparedness, vol. 7, no. 4, 2013, pp. 413-418. doi:10.1017/dmp.2013. 43.

12. Army, Marine Corps, Navy, Air Force. Multiservice tactics, techniques, and procedures for treatment of chemical agent casualties and conventional military chemical injuries. 2007 September. FM4-02.285 MCRP 4-11.1A NTRP 4-02.22 AFTTP (I) 3-2.69.

13. Derrick JI, Gomersall CD. Protecting healthcare staff from severe acute respiratory syndrome: Filtration capacity of multiple surgical masks. Journal of Hospital Infection, vol. 59, no. 4, 2005, pp. 365-368. doi:10.1016/j.jhin.2004.10. 013.

14. Leonas KK. Effect of laundering on the barrier properties of reusable surgical gown fabrics. Am J Infect Control 1998;26(5):495-501. doi:10.1016/s0196-6553(98)70022-7

15. Tammelin A, Ljungqvist B, Reinmüller B. Single-use surgical clothing system for reduction of airborne bacteria in the operating room. J Hosp Infect 2013;84(3):245-247. doi:10.1016/j.jhin.2013.03.007

16. Hospenthal DR, Green AD, Crouch HK, et al. Infection and control in deployed military medical treatment facilities. J Trauma 2011;71:S920-S298.

17. Joint Publication 3-11. Operations in chemical, biological, radiological, and nuclear environments. 2018 October 29. 